\title{
Inteligencia emocional y rendimiento académico en estudiantes universitarios de Farmacia y Bioquímica en Huancayo
}

\section{Emotional intelligence and academic performance in university students of Pharmacy and Biochemistry in Huancayo}

Iris Yone Carrasco Díaz ${ }^{1}$

Universidad Peruana Los Andes

\section{RESUMEN}

Objetivos: Determinar el grado de relación entre la inteligencia emocional y el rendimiento académico de los estudiantes de la carrera profesional de Farmacia y Bioquímica de la Universidad Peruana "Los Andes". Métodos: Es un estudio con diseño descriptivo correlacional, para ello han participado 150 estudiantes del $1^{\circ}$ al $9^{\circ}$ ciclo a quienes se les aplicaron el Inventario Emocional de BarOn, Inventario de Coeficiente Emocional (I-CE) que permitió conocer su inteligencia emocional, asimismo se recopilaron los promedios ponderados al final del semestre 2012-II de cada estudiante para medir el rendimiento académico. Resultados: Los estudiantes que presentaron una inteligencia emocional promedio $o$ adecuado fue $(X 2=183,33$ у $p=0,00) 81(54 \%)$; los que presentaron una capacidad emocional bajo son 45 (30\%); en cuanto a los componentes; intrapersonal presentaron una capacidad emocional adecuada o promedio es 95 $(63 \%)$, siendo la más alta en relación a los demás componentes; seguido de adaptabilidad 89 (59\%), manejo de estrés 86 (57\%); interpersonal 76 (51\%) y estado de ánimo 74 (49\%) quienes presentaron una inteligencia emocional promedio o adecuado; los estudiantes que alcanzaron una baja capacidad emocional fueron en los siguientes componentes: adaptabilidad 56 (37\%); interpersonal 41 (27\%); intrapersonal $36(24 \%)$; estado de ánimo $40(27 \%)$ y $32(22 \%)$ en manejo de estrés. El rendimiento académico promedio semestral es de 12,34 categorizado como regular para 98 (65\%) de estudiantes. Conclusiones: Se determinó que

1 Dra. en Administración de la Educación, investigadora de la Universidad Nacional del Centro del Perú, docente de pregrado y postgrado en las universidades Continental, y Peruana "Los Andes" 


\section{ABSTRACT}

Objectives: To determine the relationship degree between the emotional intelligence and academic performance of students from the Pharmacy and biochemistry professional career in the Peruvian university "Los Andes". Methods: It is a descriptive correlational design research. 150 students have participated from the $1^{\circ}$ to the $9^{\circ}$ cycle, on them has been applied the BarOn Emotional Inventory, emotional coefficient inventory (I-CE) which allow to know their emotional intelligence. At the same time, the weighted average was collected at the end of the semester 2012-II of each student to measure the academic performance. Results: Students who have an average or adequate emotional intelligence $(X 2=$ $183,33$ and $p=0,00)$ are $81(54 \%)$; the ones who have a low emotional capacity are $45(30 \%)$; regard to the components; the intrapersonal presents an adequate or average emotional capacity of 95 (63\%), being the highest in relation to the other components; followed by adaptability 89 (59\%), stress management $86(57 \%)$; interpersonal $76(51 \%)$ and the mood 74 (49\%) who present an adequate or average emotional capacity; the students who have a low emotional capacity were in the following components: adaptability 56 (37\%), interpersonal $41(27 \%)$, intrapersonal 36 $(24 \%)$, the mood $40(27 \%)$ and $32(22 \%)$ in stress management. The semiannual average academic performance is 12.34 categorized as regular for 98 (65\%) students. Conclusions: It was determined that there is a significant correlation between the emotional intelligence with academic performance $(r=0.403)$ $(p \leq 0.01)$. In relation to the components: intrapersonal, interpersonal, adaptability, stress management and moods also present a significant correlation $(p \leq 0.01)$ with academic performance.

Keywords: emotional intelligence, academic performance, adaptability, stress management.
INTRODUCCIÓN

El problema general fue, ¿̇cuál es la relación entre la inteligencia emocional y el rendimiento académico de los estudiantes de la carrera profesional de Farmacia y Bioquímica de la Universidad Peruana "Los Andes" Huancayo 2012?

Los problemas específicos fueron: a) ¿Cuál es la relación entre el componente intrapersonal y el rendimiento académico en estudiantes de la carrera profesional de Farmacia y Bioquímica de la Universidad Peruana "Los Andes" Huancayo 2012?; b) ¿Cuál es la relación entre el componente interpersonal y el rendimiento académico en estudiantes de la carrera profesional de Farmacia y Bioquímica de la Universidad Peruana "Los Andes" Huancayo 2012?; c) ¿Cuál es la relación entre el nivel del componente de adaptabilidad y el rendimiento académico en estudiantes de la carrera profesional de Farmacia y Bioquímica de la Universidad Peruana "Los Andes".?; d) ¿Cuál es la relación entre el nivel del componente del manejo del estrés y el rendimiento académico en estudiantes de la carrera profesional de Farmacia y Bioquímica de la Universidad Peruana "Los Andes" Huancayo 2012?; y e) ¿Cuál es la relación entre el nivel del componente estado de ánimo en general y el rendimiento académico en estudiantes de la carrera profesional de Farmacia y Bioquímica de la Universidad Peruana "Los Andes" Huancayo 2012?

El objetivo general fue determinar el grado de relación entre la inteligencia emocional y el rendimiento académico de los estudiantes de la carrera profesional de Farmacia y Bioquímica de la Universidad Peruana "Los Andes", Huancayo, 2012. Los objetivos específicos fueron determinar en los referidos estudiantes la relación de cada uno de los niveles de los componentes tales como intrapersonal, interpersonal, de adaptabilidad, de manejo de estrés, y de estado de ánimo en general, con el rendimiento académico. 
Tabla $N^{\circ}$ 1: Variables e indicadores

\begin{tabular}{|c|c|c|c|c|c|}
\hline Variable I & $\begin{array}{l}\text { Definición } \\
\text { conceptual }\end{array}$ & $\begin{array}{l}\text { Definición } \\
\text { operacional }\end{array}$ & Dimensión & Indicadores & $\begin{array}{l}\text { Escala de } \\
\text { medición }\end{array}$ \\
\hline \multirow[t]{5}{*}{$\begin{array}{l}\text { Inteligencia } \\
\text { emocional }\end{array}$} & $\begin{array}{l}\text { Es la capacidad } \\
\text { de: sentir, } \\
\text { entender, } \\
\text { controlar y } \\
\text { modificar } \\
\text { estados anímicos } \\
\text { propios y ajenos. } \\
\text { Goleman } \\
\text { (1996). }\end{array}$ & $\begin{array}{l}\text { La inteligencia } \\
\text { emocional } \\
\text { se conocerá } \\
\text { aplicando el } \\
\text { Test de Ice Bar- } \\
\text { On medida } \\
\text { por } 5 \text { escalas } \\
\text { individuales y } 15 \\
\text { sub escalas con } \\
\text { un total de } 133 \\
\text { ítems. }\end{array}$ & Intrapersonal & $\begin{array}{l}\text { Comprensión } \\
\text { Emocional de sí } \\
\text { Mismo. (CM) } \\
\text { Asertividad (AS). } \\
\text { Autoconcepto. } \\
\text { (AC) } \\
\text { Autorrealización } \\
\text { (AR) }\end{array}$ & $\begin{array}{l}\text { Intervalo } \\
130 \text { y más: } \\
\text { Capacidad } \\
\text { emocional muy } \\
\text { desarrollada } \\
115 \text { a 129: } \\
\text { Buena capacidad } \\
\text { emocional. } \\
86 \text { a 114: } \\
\text { Capacidad } \\
\text { emocional } \\
\text { adecuada } \\
\text { Promedio. } \\
70 \text { a } 85 \text { : } \\
\text { Capacidad } \\
\text { emocional por } \\
\text { debajo del } \\
\text { promedio. } \\
69 \text { y menos: } \\
\text { Capacidad } \\
\text { emocional muy } \\
\text { baja. }\end{array}$ \\
\hline & & & Intrapersonal & \begin{tabular}{l}
\multicolumn{1}{c}{ Empatía } \\
Relaciones \\
Interpersonales \\
(RI) \\
Responsabilidad \\
Social (RS)
\end{tabular} & Intervalo \\
\hline & & & Adaptabilidad & $\begin{array}{l}\text { Solución de } \\
\text { problemas (SP) } \\
\text { Prueba de la } \\
\text { realidad (PR) } \\
\text { Flexibilidad (FL) }\end{array}$ & Intervalo \\
\hline & & & $\begin{array}{l}\text { Manejo de } \\
\text { Estrés }\end{array}$ & $\begin{array}{l}\text { Tolerancia al } \\
\text { estrés (TE) } \\
\text { Control de los } \\
\text { impulsos (Cl) }\end{array}$ & Intervalo \\
\hline & & & $\begin{array}{l}\text { Estado de } \\
\text { ánimo en } \\
\text { General }\end{array}$ & $\begin{array}{l}\text { Felicidad (FE) } \\
\text { Optimismo (OP) }\end{array}$ & Intervalo \\
\hline
\end{tabular}

La hipótesis general fue: existe relación significativa entre la inteligencia emocional y el rendimiento académico de los estudiantes de la carrera profesional de Farmacia y Bioquímica de la Universidad Peruana "Los Andes" Huancayo 2012. Las hipótesis específicas fueron, a) El componente intrapersonal está relacionado significativamente con el rendimiento académico de los estudiantes de la carrera profesional de Farmacia y Bioquímica de la Universidad Peruana "Los Andes"; b) El componente Interpersonal está relacionado significativamente con el rendimiento académico de los estudiantes de la carrera profesional de Farmacia y Bioquímica de la Universidad Peruana "Los Andes"; c) El componente de adaptabilidad está 
Tabla $N^{\circ}$ 2: Variables e indicadores

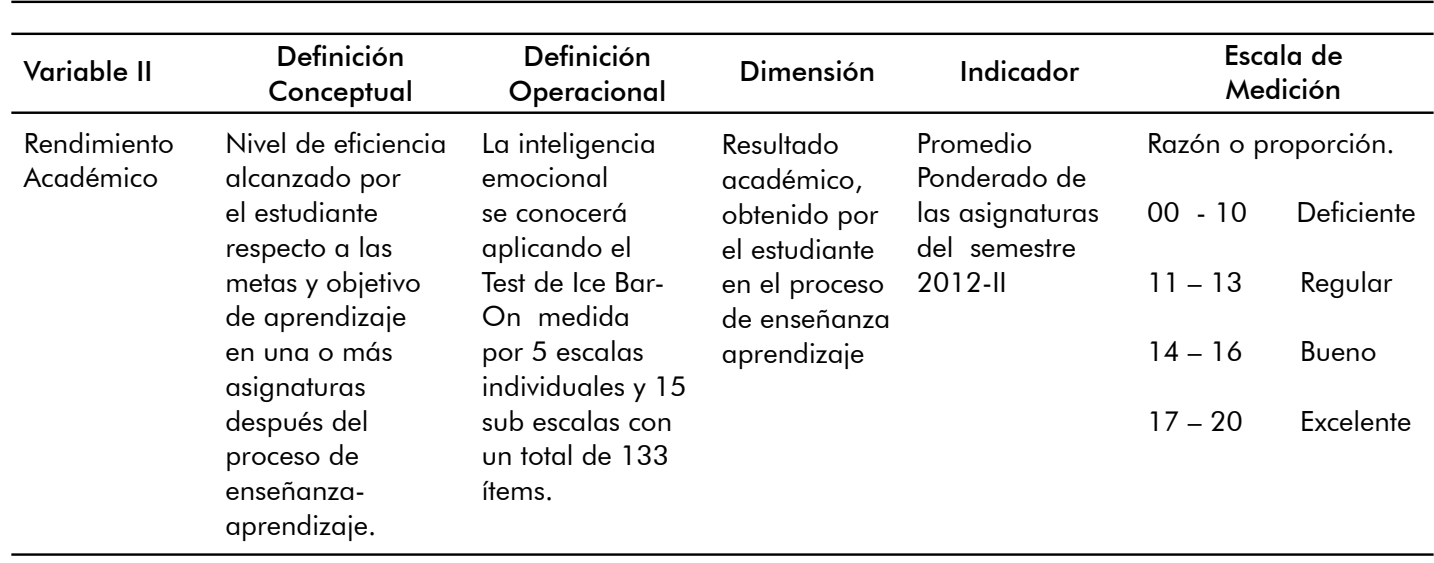

relacionado significativamente con el rendimiento académico de los estudiantes de la carrera profesional de Farmacia y Bioquímica de la Universidad Peruana "Los Andes"; d) El componente Manejo de estrés está relacionado significativamente con el rendimiento académico de los estudiantes de la carrera profesional de Farmacia y Bioquímica de la Universidad Peruana "Los Andes"; e) El componente estado de animo en general está relacionado significativamente con el rendimiento académico de los de la carrera profesional de Farmacia y Bioquímica de la Universidad Peruana "Los Andes".

Definiciones importantes del estudio: Salovey y Mayer (1) define "Inteligencia Emocional" como la habilidad para percibir, valorar y expresar emociones con exactitud, la habilidad de acceder y/o generar sentimientos que faciliten el pensamiento; la habilidad para comprender emociones y conocimiento emocional; la habilidad para regular emociones que promuevan el crecimiento intelectual $y$ emocional.

Goleman (2), aportó al mundo de la inteligencia emocional investigaciones acerca del cerebro emocional, donde hace reflexionar sobre la particularidad de que los hombres, a pesar de ser el animal racional por excelencia, se dejen llevar por estímulos emocionales irracionales. No obstante, señala que estas conductas emocionales puedan ser controladas, aunque para ello habrá que moldear esos hábitos.

\section{MATERIAL Y MÉTODOS}

El presente estudio es una investigación básica, tiene como propósito recoger información de la realidad para enriquecer el conocimiento teórico - científico, orientándonos al descubrimiento de principios y leyes, según Sánchez $\mathrm{H}$. y Reyes C. (3). El diseño utilizado es descriptivo correlacional compuesto por una muestra con dos medidas.

\section{Población - muestra}

La población estuvo constituida por 573 estudiantes de la carrera profesional de Farmacia y Bioquímica del semestre 2012II desde el I al IX ciclo.

La muestra fue de 150 estudiantes; se calculó utilizando el $95 \%$ de confianza y con un margen de error de 0,03.

El tipo de muestreo utilizado fue el estratificado por afijación proporcional en relación a los ciclos, así como el muestreo aleatorio simple dentro de cada ciclo.

Tabla Nº 3: Técnicas e instrumentos

\begin{tabular}{cc}
\hline Técnica & Instrumentos \\
\hline Encuesta & Cuestionario - Inventario \\
& Emocional De BarOn (I-CE) \\
Promedio ponderado & Boleta de notas. \\
\hline
\end{tabular}




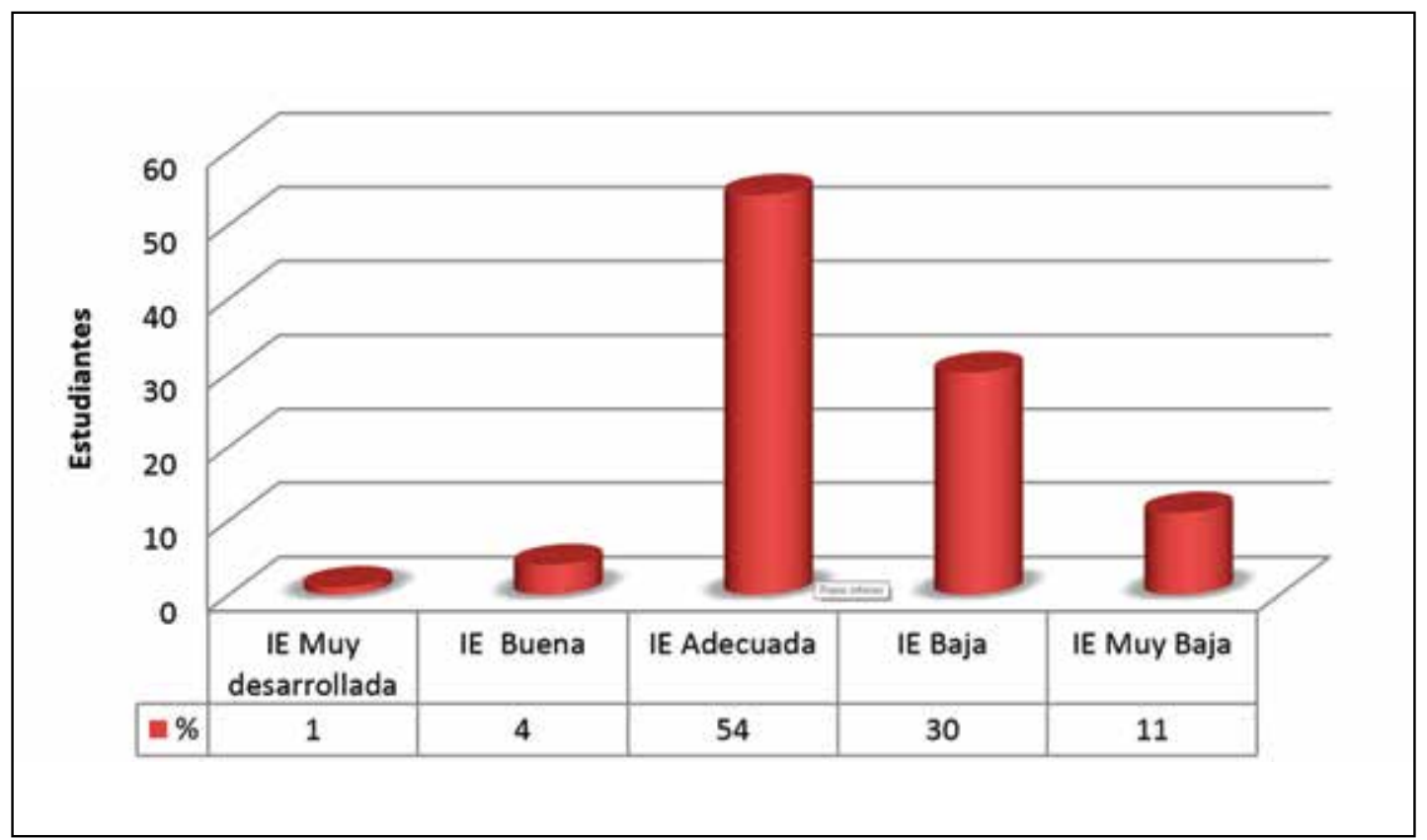

Figura $\mathrm{N}^{\circ}$ 1: Niveles del coeficiente de inteligencia emocional total de los estudiantes de la carrera profesional de Farmacia y Bioquímica de la Universidad Peruana "Los Andes", Huancayo

\section{Modelo de inteligencia emocional y social de BarOn}

BarOn (4) cit. por Ugarriza N. (5) define a la inteligencia emocional como, un conjunto de habilidades personales, emocionales $y$ sociales $y$ de destrezas que influyen en nuestra habilidad para adaptarnos y enfrentar las demandas y presiones del medio. Como tal, nuestra inteligencia no cognitiva es un factor importante en la determinación de la habilidad para tener éxito en la vida, influyendo directamente en el bienestar general y en la salud emocional.

Sobre la base de este concepto, BarOn construye el inventario de cociente emocional (I-CE), siendo necesario presentar el marco de trabajo conceptual de este. El modelo de BarOn considera cinco componentes y sus respectivos subcomponentes que se relacionan lógica y estadísticamente que son evaluadas por el BarOn Emotional Quotient Inventory (BarOn EQ-I).Este inventario genera un cociente emocional y cinco cocientes emocionales compuestos basados en las puntuaciones de 15 subcomponentes.

Sobre el Rendimiento Académico, Adell, explica al rendimiento, como: “Un sinónimo de beneficio, que consiste en alcanzar el mejor resultado en menor tiempo y esfuerzo posible."

En la Universidad Peruana "Los Andes", con Resolución $N^{\circ}$ 0260-CF-FCC.SSUPLA-2011, se aprueba el cuadro de equivalencias de calificaciones para el rendimiento académico, establecidas por la Oficina de Desarrollo Académico de la Universidad, conforme se detalla: De 00-10 (Deficiente), 11-13(Regular), 14-16(Bueno), 17-20(Excelente), (Anexo № 2).

\section{RESULTADOS}

\section{Resultados de inteligencia Emocional}

Como puede observarse en la figura $\mathrm{N}^{\circ}$ 1 se obtienen los resultados globales obtenidos mediante el inventario de Bar-On para los niveles de inteligencia emocional, el cociente o nivel de inteligencia emocional total que predomina de manera acentuada 


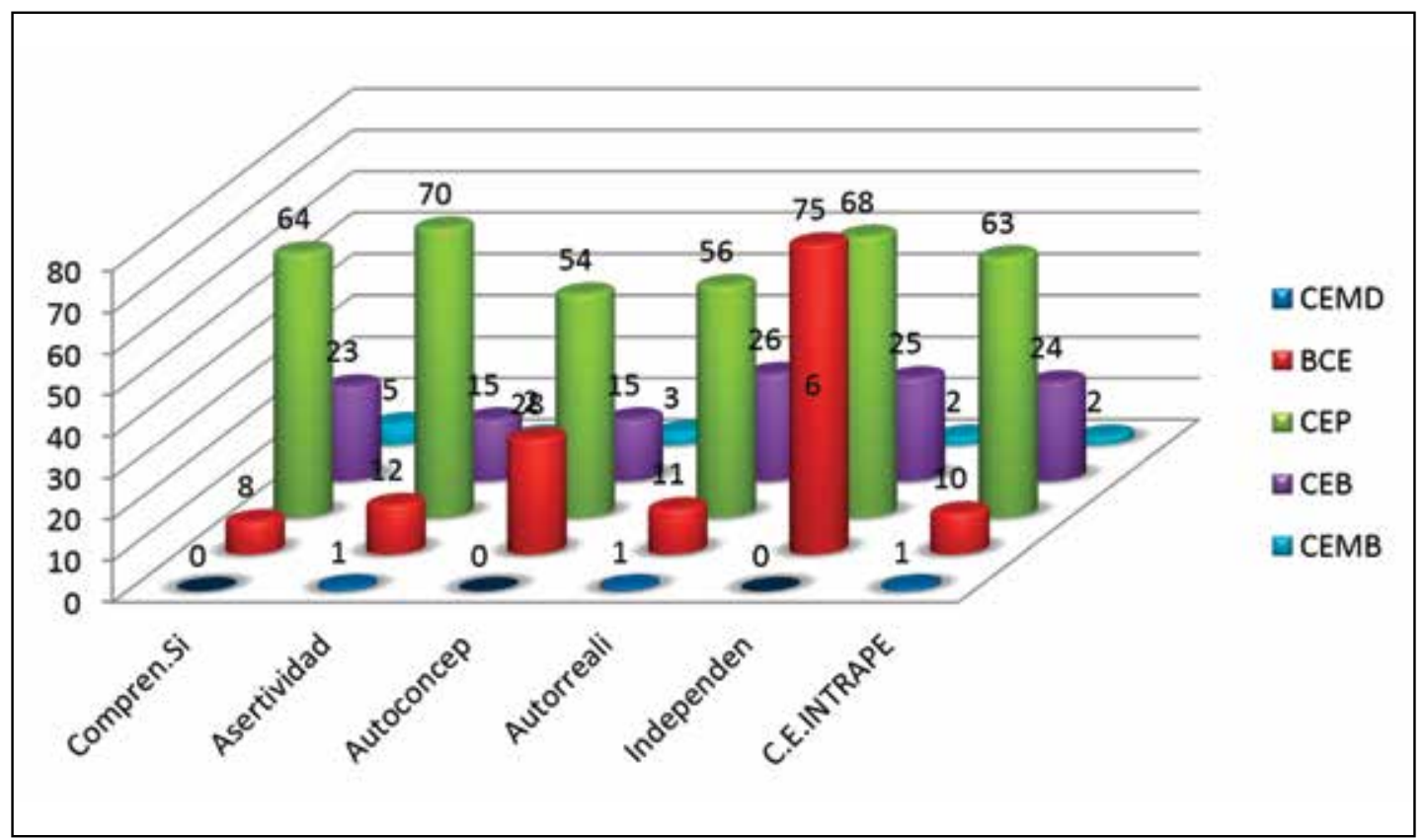

Figura $\mathrm{N}^{\circ}$ 2: Niveles del área intrapersonal de la inteligencia emocional en estudiantes de la carrera profesional de Farmacia y Bioquímica de la Universidad Peruana "Los Andes" Huancayo

es el adecuado o promedio $54 \%$, un $4 \%$ se ubica en el nivel bueno, un $30 \%$ con un nivel bajo y $11 \%$ muy bajo. Para probar si los niveles totales son diferenciados se utilizó la prueba no paramétrica de Chi cuadrado de Pearson, con 4 G.L. y X2=183,33 y $p=0,00$ lo que indica que estadísticamente existe una diferencia significativa entre los niveles totales de inteligencia emocional.

En la figura $\mathrm{N}^{\circ} 2$, en relación al componente intrapersonal se aprecia que globalmente un $63 \%$ (95) lograron puntajes con capacidad emocional adecuado; es decir, son estudiantes que se sienten bien acerca de sí mismos y positivos en lo que se están desempeñando; el $1 \%$ con capacidad emocional muy desarrollada; un $24 \%$ (36) con un nivel bajo y un $2 \%$ con un nivel muy bajo.

Al analizar los niveles en los que se ubican los sujetos de estudio en cada uno de los cinco subcomponentes del intrapersonal apreciamos que: En la habilidad de ser asertivos, capaces de expresar sus sentimientos, creencias y pensamientos sin dañar los sentimientos de los demás, defender sus derechos de una manera no destructiva, un $70 \%$ (105) se ubica en el nivel de capacidad emocional adecuado o promedio, $12 \%$ (18) se ubican en el nivel de bueno, y $15 \%$ (22) presentan el nivel bajo y un $5 \%$ (8) con un nivel muy bajo por lo que requieren mejora.

En la capacidad emocional, que implica comprensión emocional de sí mismo, reconociendo sus propios sentimientos y emociones saber que los ocasionó encontramos con un nivel adecuado 0 promedio $64 \%(96)$, con un nivel bueno $8 \%$ (12) y con $23 \%$ (34) con un nivel bajo y un $5 \%$ (8) con un nivel muy bajo. En cuanto a la habilidad de autorrealización, que caracteriza a los individuos que saben lo que quieren o sus metas adónde se dirigen y por qué, capaces de realizar lo que realmente pueden y quieren, disfrutando de hacerlo, encontramos a un $56 \%$ (84) con un nivel promedio o adecuado; $11 \%$ (17) los que se ubicaron en el nivel de capacidad emocional bueno; $1 \%$ (17) con un nivel muy desarrollado; así mismo, encontramos un $26 \%$ (39) con un nivel bajo y 6\% (9) están en el nivel muy bajo.

En cuanto al subcomponente de independencia, personas que confían en sí mismas, autónomas e independientes en su forma de pensar y actuar, encontramos con un $68 \%$ (103) con capacidad emocional 


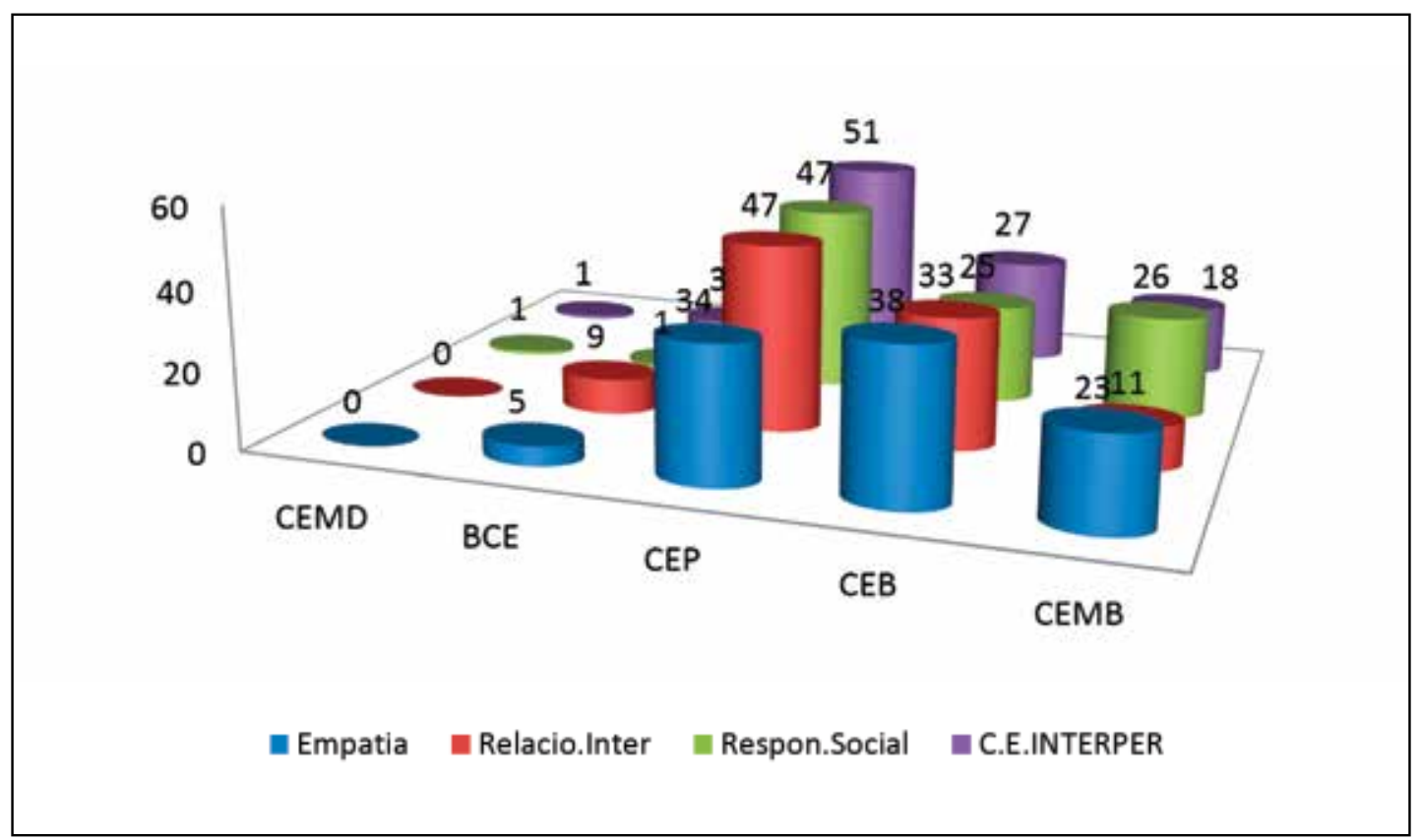

Figura $\mathrm{N}^{\circ}$ 3: Niveles del área interpersonal de la inteligencia emocional en estudiantes de la carrera profesional de Farmacia y Bioquímica de la Universidad Peruana "Los Andes" Huancayo 2012-II

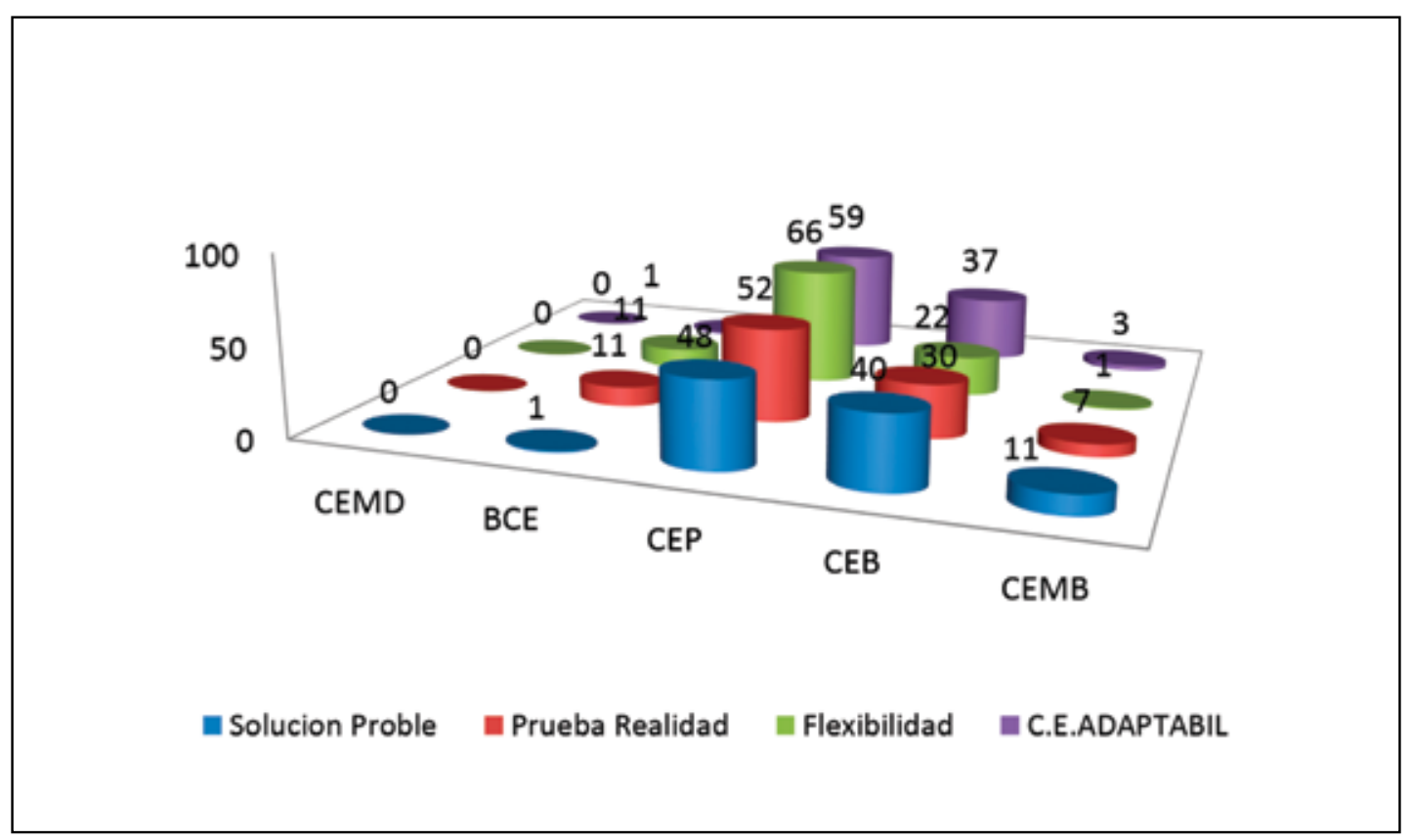

Figura $\mathrm{N}^{\circ}$ 4: Niveles del área adaptabilidad de la inteligencia emocional en estudiantes de la carrera profesional de Farmacia y Bioquímica de la Universidad Peruana "Los Andes" Huancayo- 2012-I

adecuada, con un $5 \%$ (7) las que se ubicaron en el nivel de capacidad bueno, asimismo con un $25 \%$ (37) lo están en el nivel bajo y $2 \%(3)$ se ubicaron en el nivel muy bajo.

Observamos que en el componente interpersonal de acuerdo a la figura $\mathrm{N}^{\circ} 3$ el $51 \%$ (76) los que muestran un nivel de inteligencia emocional promedio (CEP); es decir, son estudiantes que cuentan con buenas habilidades sociales; 27 (41\%) se ubican en el nivel bajo (CEB); 27 (18\%) en muy bajo; sólo 4 (3\%) lograron puntajes que los ubican en el nivel bueno y muy alto el 1\% (2). Apreciamos que de 


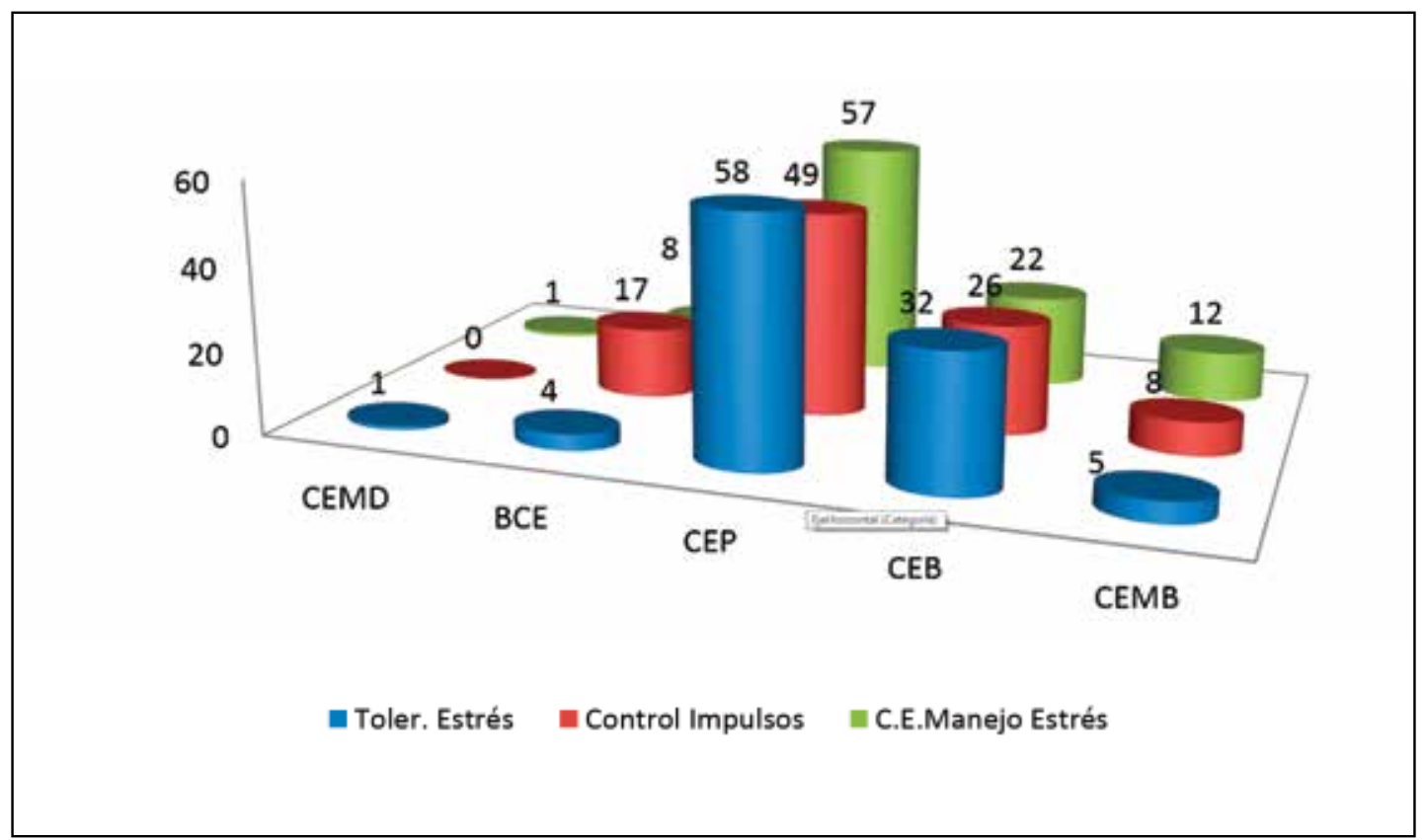

Figura $\mathrm{N}^{\circ}$ 5: Niveles del área Manejo de estrés de la Inteligencia Emocional en estudiantes de la carrera profesional de Farmacia y Bioquímica de la Universidad Peruana "Los Andes" Huancayo- 2012-II.

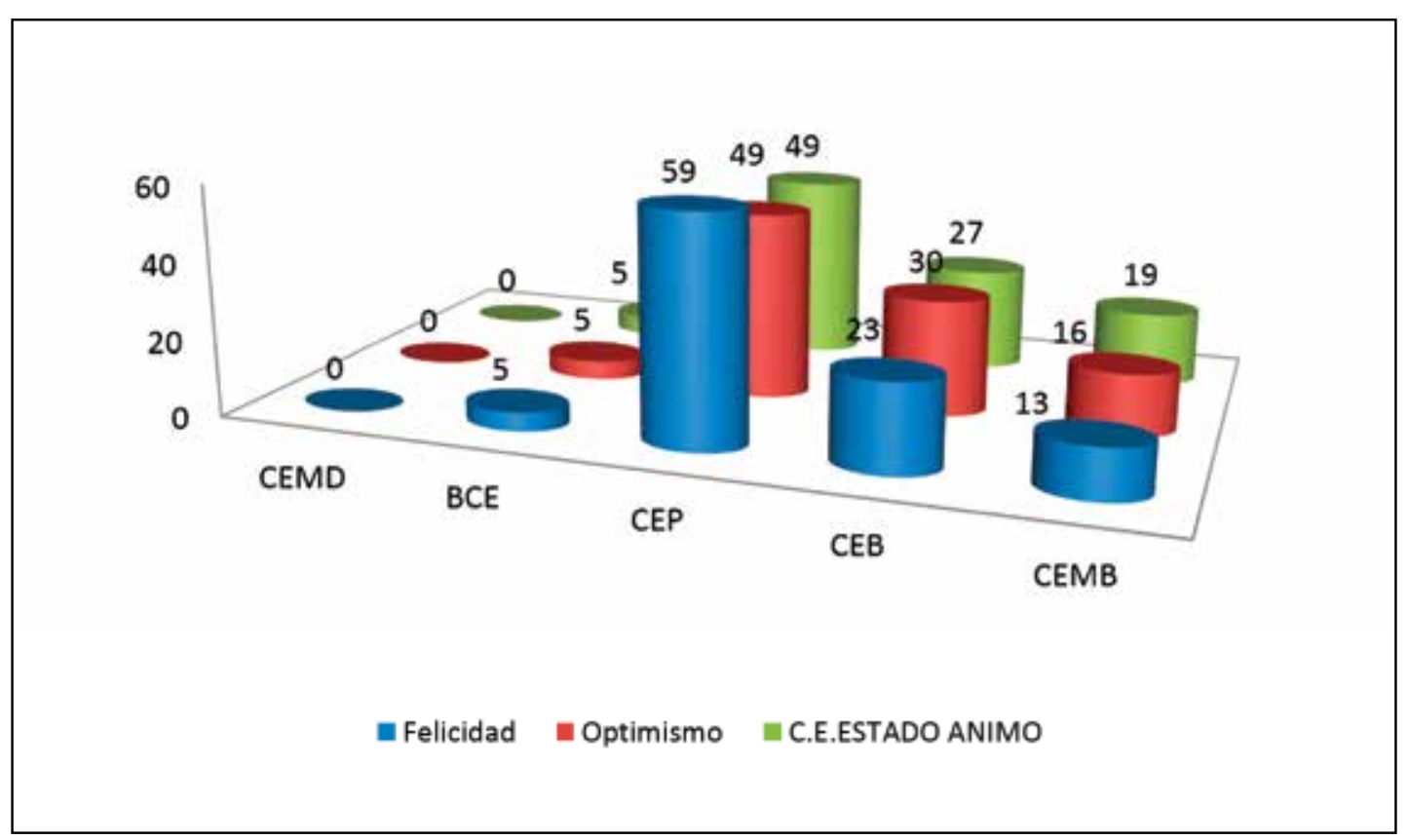

Figura $\mathrm{N}^{\circ}$ 6: Niveles del área estado de animo de la inteligencia emocional en estudiantes de la carrera profesional de Farmacia y Bioquímica de la Universidad Peruana "Los Andes" Huancayo 2012-II.

los tres subcomponentes que integran el componente interpersonal, es en el de responsabilidad social $47 \%$ (72), donde se obtuvo el puntajes más alto; seguido del subcomponente relaciones interpersonales $47 \%$ (71), quienes obtuvieron un nivel de inteligencia promedio; en caso empatía el
$38 \%$ (57), obtuvieron un coeficiente bajo, estudiantes que no aprecian los sentimientos de los demás; seguido de un 34\% (51) con un coeficiente promedio.

En la figura $\mathrm{N}^{\circ} 4$ observamos que en el componente adaptabilidad el $59 \%$ (89) se 


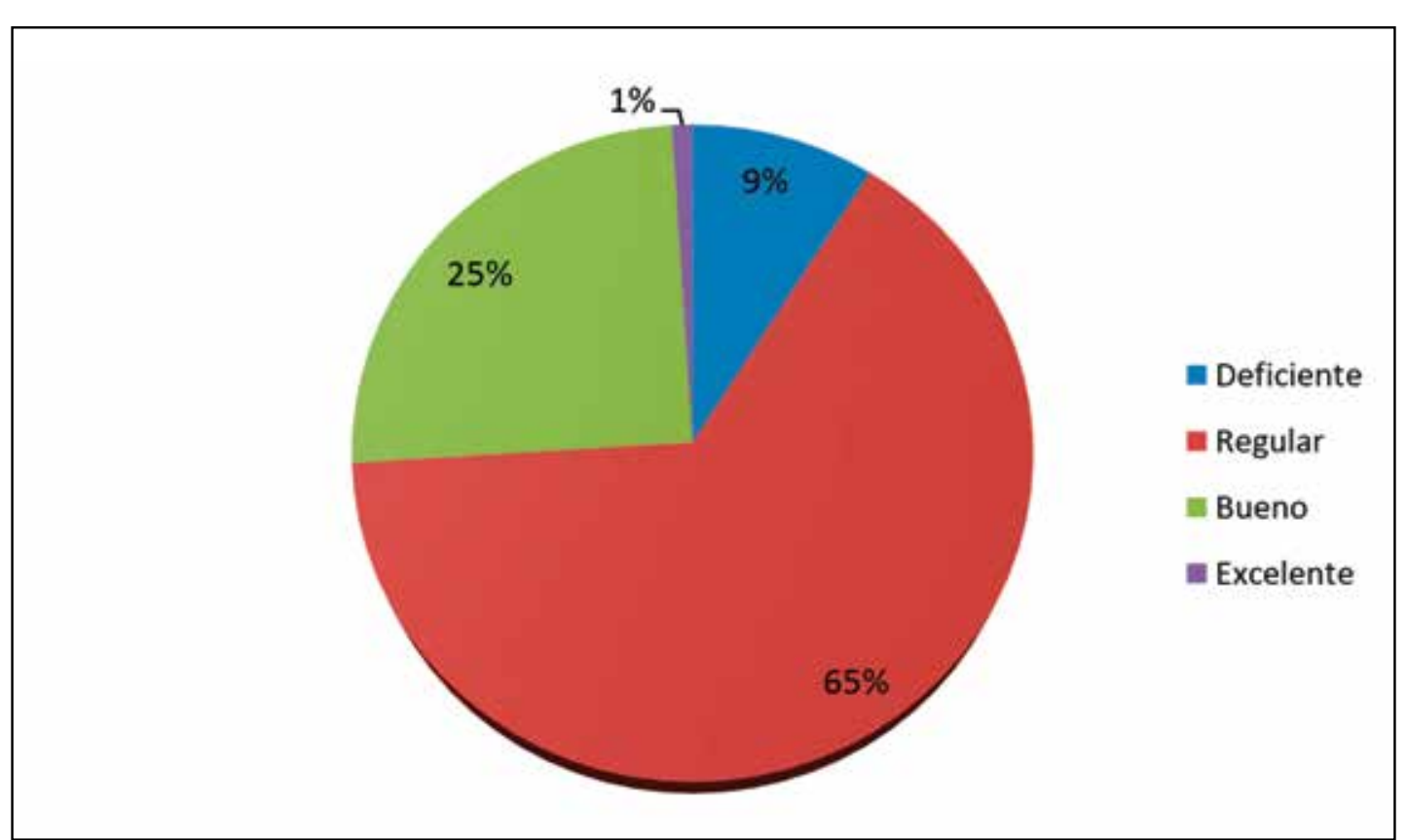

Figura $N^{\circ}$ 7: Rendimiento Académico de los estudiantes de la carrera profesional de Farmacia y Bioquímica de la Universidad Peruana "Los Andes" Huancayo 2012-II

ubican en el nivel promedio o adecuado; es decir, son estudiantes que se adecuan a las exigencias del entorno; $37 \%$ (56) se ubican en el nivel bajo (CEB) y muy bajo el $3 \%(4)$; solo el $1 \%(1)$ tiene un nivel bueno, asimismo; en cuanto al subcomponente flexibilidad (tolerantes a distintas ideas) el $66 \%(99)$ presenta un coeficiente promedio; un $22 \%$ (33) un coeficiente bajo, muy bajo $1 \%$ (1) y un $11 \%$ (17) un nivel bueno. En relación al subcomponente prueba de la realidad un $52 \%$ (78) presenta un nivel promedio y un $30 \%$ (45) nivel bajo y muy bajo un $7 \%$ (11), y un buen nivel el $11 \%$ (16). En relación a solución de problemas el $48 \%$ (72)presenta un nivel promedio; bajo el $40 \%$ (60) y muy bajo el $11 \%$ (17).

En relación a la figura $\mathrm{N}^{\circ} 5$, manejo de estrés, área que indica cuanto puede ser capaz una persona de resistir la tensión sin perder el control el 57\% (86) presenta un nivel promedio; un $22 \%$ (32) un nivel bajo; $12 \%$ (18) muy bajo y un $8 \%$ (12) un nivel bueno. En relación al subcomponente control de impulsos el 49\% (74) presenta un coeficiente promedio; $26 \%$ (39) bajo y muy bajo; el $8 \%$ (12) y buen coeficiente emocional el $17 \%$ (25). En relación a tolerancia al estrés el $58 \%$ (87) presenta un nivel promedio, el $32 \%$ (49) bajo y muy bajo $5 \%(7)$, el $4 \%(6)$ buen cociente emocional y el $1 \%(1)$ el nivel muy desarrollado.

En relación a la figura $\mathrm{N}^{\circ} 6$, en cuanto al componente estado de ánimo que es la capacidad del estudiante para disfrutar de la vida así como de la visión que tiene de la misma y el sentimiento de contento en general. Observamos que el 49\% (74) tiene un nivel adecuado; el $27 \%$ (40) bajo; muy bajo 19\% (28); el 5\% (8) presenta un buen nivel. En cuanto al subcomponente optimismo el $49 \%$ (73) tiene un nivel promedio; el 30\% (46) bajo; muy bajo el $16 \%(24)$ y un $5 \%$ (7) un nivel bueno. En relación al subcomponente felicidad el $59 \%$ (89) presenta un nivel adecuado; el 23\% (34) nivel bajo; el 13\% (19) muy bajo y solo un $5 \%$ (8) buen cociente emocional.

\section{Resultados del rendimiento académico}

En relación a la figura $\mathrm{N}^{\circ} 7$, el rendimiento académico de los estudiantes de la carrera profesional de Farmacia y Bioquímica en el semestre 2012-II tienen como promedio 12.34. Así mismo, se observa que el $65 \%$ 


\section{Resultados de correlaciones de la inteligencia emocional general y sus componentes}

Tabla $N^{\circ}$ 4: Correlaciones con $r$ de Pearson entre la inteligencia emocional y rendimiento académico de los estudiantes de la carrera profesional de Farmacia y Bioquímica de la Universidad Peruana "Los Andes"

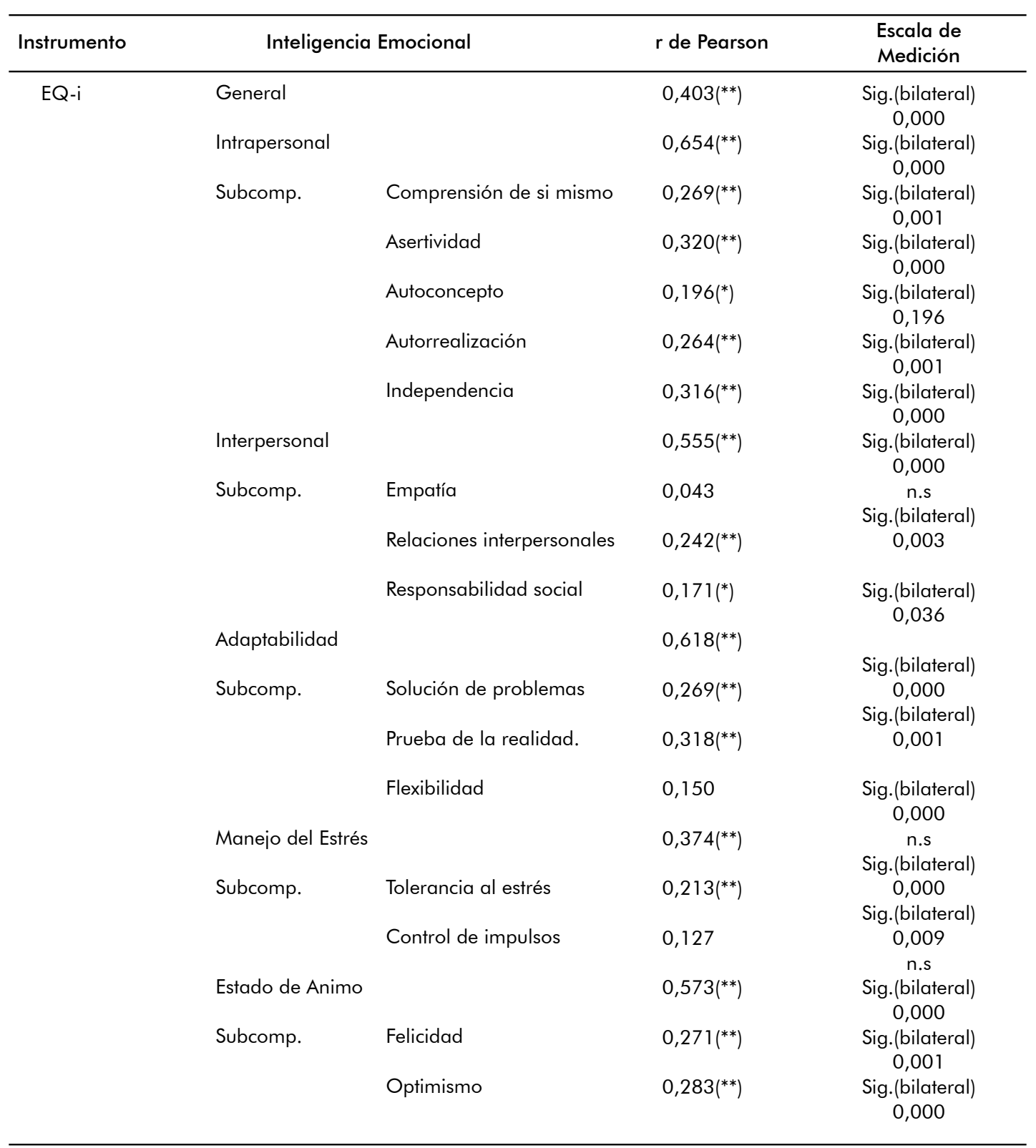

Correlación significativa ${ }^{*} \mathrm{p} \leq 0.05{ }^{* *} \mathrm{p} \leq 0.01 ;$ n.s: no significativa

(8) de estudiantes son regulares (11-13); el $25 \%$ (37) buenos (14-16); deficientes (0010) el $9 \%(14)$ y solo el $1 \%(1)$ excelentes (17-20).

\section{DISCUSIÓN}

Es innegable que los individuos se adaptan o afrontan las demandas y presiones del ambiente de forma distinta. A cualquier edad y en cualquier cultura tienen y utilizan 
diversas competencias, habilidades $y$ destrezas para manejar el estrés, establecer relaciones interpersonales, dirigir sus emociones y estados de ánimo, adaptándose y ajustándose al medio a fin de conseguir resultados académicos óptimos.

En la tarea de optimizar la calidad del proceso educativo, reviste particular importancia reflexionar sobre una de las variables que influyen en el rendimiento académico de los estudiantes: su inteligencia emocional. Aquí mostramos la relación entre la inteligencia emocional y el rendimiento académico.

En los resultados observamos en primer lugar que el nivel de inteligencia emocional global o total (CET) de los estudiantes investigados se encuentra ubicado mayoritariamente en el nivel promedio (54\%), (tabla $\mathrm{N}^{\circ} 1$ ) lo que significa una capacidad emocional adecuada, $\quad(X 2=183,33) \quad(p=0,000)$ típicamente saludable, la misma que les permitirá manejar las exigencias del entorno (4) controlando sus emociones, comunicándose de forma activa y proyectando una actitud optimista ante la vida. El $4 \%$ se encuentra en el nivel bueno, lo que revela una buena capacidad emocional, se siente bien consigo mismo y generalmente tiene éxito para relacionarse con las demás personas. De otro lado, un $30 \%$ (45) de los estudiantes se ubican en el nivel bajo, y $11 \%$ muy bajo, requiriendo apoyo para mejorar algunas competencias y habilidades particulares, a fin de que pueda enfrentar las exigencias del futuro.

Estos resultados son corroborados de alguna manera por Villacorta E. (6), que al estudiar la "inteligencia emocional y rendimiento académico en estudiantes de Medicina Humana de la Universidad Nacional de la Amazonía Peruana." encontraron que la media de los sujetos de la muestra se ubicaron en el nivel promedio, seguido por el alto o muy alto (CEA) y finalmente por el bajo (CEB). Estos dos últimos difieren de nuestra investigación.

Al realizar el análisis de la inteligencia emocional por componentes de los estudiantes de la muestra (tablas $\mathrm{N}^{\circ} 2, \mathrm{~N}^{\circ}$
$4, \mathrm{~N}^{\circ} 5, \mathrm{~N}^{\circ} 3$ y $\mathrm{N}^{\circ} 6$ ), encontramos que, en promedio, las competencias referidas a intrapersonal (63\%) ocupan los niveles promedios, son estudiantes que se sienten bien acerca de sí mismos, personas capaces de expresar abiertamente sus sentimientos, confían en la realización de sus ideas. seguidas muy de cerca por las de adaptabilidad (59\%), estudiantes que lidian de manera efectiva las situaciones problemáticas, y manejo de estrés (57\%), donde se evidencia cuanto pueden ser capaces de resistir a la tensión sin perder el control, llegando a ser calmados y trabajar bajo presión, estando luego las capacidades interpersonal (51\%) y estados de ánimo (49\%), sujetos con tendencia para disfrutar la vida, discrepando con Villacorta E. (6) quien en su investigación encuentra mayores fortalezas en la dimensión estado de ánimo general (CEAG), seguida por la de adaptabilidad (CAD), continuando la intrapersonal (CIA) y manejo de estrés $(\mathrm{CME})$, ubicándose al final la interpersonal (CIE).

Se evidencia una marcada tendencia de los sujetos del estudio, que se sienten bien acerca de sí mismos, que se sienten positivos en lo que están desempeñando; así mismo, son personas que se adecúan a las exigencias del entorno, resistir al estrés sin "desmoronarse" o sin perder el control-, trabajar bajo presión, sentirse bien consigo mismo y con lo que hacen en la vida, comprendiendo, interactuando y relacionándose muy bien con los demás. Así mismo, se evidencia una tendencia de los sujetos para disfrutar de la vida tener una perspectiva positiva de ella, sentirse contentos en general. Son características que los muestran como personas optimistas, satisfechas con su propia vida, realistas y hábiles para reconocer, definir e intentar resolver problemas, capaces de adecuar sus emociones, sentimientos, pensamientos y comportamientos a situaciones y condiciones cambiantes; con habilidades para ser tolerantes al estrés y capaces de interaccionar positivamente con los demás.

En relación al rendimiento académico de los estudiantes, observamos que el $65 \%$ 
de los alumnos de la carrera profesional de Farmacia y Bioquímica (tabla $\mathrm{N}^{\circ} 7$ ) se sitúan en la categoría de calificación regular, mientras que un $25 \%$ se ubica en la categoría bueno, siendo la media semestral de 12,34. En la investigación de Villacorta E. (6) el 92,2\% de los alumnos de la Facultad de Medicina Humana de la UNAP se sitúa en la categoría de calificación regular, mientras que un $7,8 \%$ se ubica en la categoría de bueno.

En cuanto a la hipótesis general: "existe una relación significativa entre la inteligencia emocional y el rendimiento académico" se han encontrado resultados de correlación significativos $(r=0,403, p \leq 0,01) \quad$ (tabla $N^{\circ}$ 8) lo que confirma la hipótesis. Estos resultados corroboran los estudios mencionados en la introducción como el de Valdivia J (7); Pérez N (8); Guerra J, et al (9); López O (10); Condolo Z y Alexis J (11); Villacorta, E. (6) Bazán (12); así mismo confirman las teorías de Sternberg (13) y Goleman (2), discrepando con Jaimes A (14), quien investigó: "La Inteligencia emocional y rendimiento académico en estudiantes de la Facultad de Educación y Humanidades de la Universidad Alas Peruanas" quien concluye que no existe una relación significativa entre ambas variables.

En cuanto a la hipótesis específica:"existe una relación significativa entre la inteligencia emocional intrapersonal y el rendimiento académico", se han encontrado resultados de correlación significativos $(r=0,654$; $p \leq 0,01$ ), así mimo en sus subcomponentes, comprensión de sí mismo $(r=0,269 ; p \leq 0,01)$; asertividad $(r=0,320 ; p \leq 0,01)$ : autoconcepto $(r=0,320 ; \quad p \leq 0,05) ; \quad$ autorrealización $(r=0,264 ; \quad p \leq 0,01) ; \quad$ e independencia $(r=0,316 ; \quad p \leq 0,05)$; lo que confirma la hipótesis.

En cuanto a la hipótesis específica:"existe una relación significativa entre la inteligencia emocional interpersonal y el rendimiento académico"; se han encontrado resultados de correlación significativos $(r=0,555$; $\mathrm{p} \leq 0,01)$, así mismo en sus subcomponentes, relaciones interpersonales $(r=0,269$; $p \leq 0,01)$; responsabilidad social $(r=0,171$; $p \leq 0,05)$ lo que confirma la hipótesis, estos resultados corroboran los estudios mencionados en la introducción como el de Flores A (15); López O (16); Villacorta E (6).

En cuanto a la hipótesis específica:"existe una relación significativa entre la inteligencia emocional adaptabilidad y el rendimiento académico"; se han encontrado resultados de correlación significativos $(r=0,618 ; p \leq 0,01)$; así mismo, en sus subcomponentes: solución de problemas $(r=0,269 ; p \leq 0,01)$; prueba de la realidad $(r=0,318 ; p \leq 0,01)$, lo que confirma la hipótesis, estos resultados corroboran los estudios mencionados en la introducción como el de Flores A (15); López O (16); Villacorta J (6).

En cuanto a la hipótesis específica: "existe una relación significativa entre la inteligencia emocional manejo de estrés y el rendimiento académico"; se han encontrado resultados de correlación significativos $(r=0,374 ; \quad p \leq 0,01) ; \quad$ así mismo, en sus subcomponentes: tolerancia al estrés $(r=0,213 ; p \leq 0,01)$; lo que confirma la hipótesis, estos resultados corroboran los estudios mencionados en la introducción como el de Flores A (15); Villacorta E (6), Jaimes A (14).

En cuanto a la hipótesis específica:"existe una relación significativa entre la inteligencia emocional estado de ánimo y el rendimiento académico"; se han encontrado resultados de correlación significativos $(r=0,573 ; \quad p \leq 0,01)$, así mismo en sus subcomponentes, felicidad $(r=0,271 ; p \leq 0,01)$; optimismo $(r=0,283$; $p \leq 0,01)$; lo que confirma la hipótesis, estos resultados corroboran los estudios mencionados en la introducción como el de López O (16); Jaimes A (14).

Los subcomponentes de inteligencia emocional que se deben tratar para mejorarlos de acuerdo al presente estudio, en los estudiantes de la Carrera Profesional de Farmacia y Bioquímica son: empatía, flexibilidad y control de impulsos, subcomponentes que si no mejoran traería como consecuencia equipos humanos 
infrautilizados y producciones mediocres comparados con su potencial.

La inteligencia emocional será el recurso más importante del siglo XXI para sobrevivir a una época de cambio permanente, básica para incrementar el rendimiento profesional, bienestar personal y especialmente profesiones complejas que hoy ofertan las universidades las que incluyen relaciones personales como centro y principal motor de la actividad académica.

Asimismo, las relaciones laborales han cambiado sustancialmente a raíz de la crisis financiera, global y económica, las personas se enfrentan a situaciones nunca conocidas hasta ahora como son el aumento de la carga de trabajo y una mayor inestabilidad laboral ante la creciente incertidumbre en la que se encuentran empresas y trabajadores, es así que este estudio aplicado al mundo de las relaciones laborales cobrará mayor importancia y protagonismo ya que permitirá que se planteen programas de desarrollo de habilidades emocionales para futuros líderes y directivos

Esta investigación contribuirá a que se evidencie cada subcomponente débil del cociente emocional de los sujetos de estudio y a través de los resultados, gestionar estrategias que posibiliten mejorar el rendimiento académico a través del desarrollo de la inteligencia emocional lo que permitirá al estudiante enfrentar con éxito las exigencias del entorno.

De acuerdo a la investigación realizada se puede concluir en lo siguiente:

Al relacionar la inteligencia emocional con el rendimiento académico de los estudiantes de la carrera profesional de Farmacia y Bioquímica de la Universidad Peruana Los Andes, se halló una correlación significativa $(p \leq 0,01)$, lo que significa que a mayor inteligencia emocional mayor rendimiento académico.

Asímismo se encontró correlación significativa $(p \leq 0,01)$ del componente intrapersonal con el rendimiento, académico de los estudiantes de la carrera profesional de Farmacia y Bioquímica de la Universidad Peruana Los Andes y de los subcomponentes comprensión emocional de sí mismo, asertividad, autorrealización, independencia $(p \leq 0,01)$ y autoconcepto $(p \leq 0,05)$, lo que significa que a mayor nivel del componente Intrapersonal y de sus subcomponentes mayor será el rendimiento académico.

En relación al componente interpersonal con el rendimiento académico de los estudiantes de la carrera profesional de Farmacia y Bioquímica de la Universidad Peruana Los Andes, se encontró que existe correlación significativa $(p \leq 0,01)$ y de los subcomponentes; relaciones interpersonales $(p \leq 0,01)$ y realidad social $(p \leq 0,05)$ lo que significa que a mayor nivel del componente interpersonal y de sus subcomponentes mayor será el rendimiento académico, a excepción del subcomponente empatía que sus resultados fueron no significativos $(p \geq 0,05)$.

En relación al componente adaptabilidad con el rendimiento académico se encontró que existe correlación significativa $(p \leq 0,01)$, así mismo de los subcomponentes; solución de problemas y prueba de la realidad, $(p \leq 0,01)$ lo que significa que a mayor nivel del componente Adaptabilidad y de sus subcomponentes mayor será el rendimiento académico de los estudiantes de la carrera profesional de Farmacia y Bioquímica de la Universidad Peruana Los Andes, a excepción del subcomponente flexibilidad cuyos resultados fueron no significativos $(p \geq 0,05)$.

En relación al componente manejo del estrés con el rendimiento académico se encontró que existe correlación significativa $(p \leq 0,01)$, así como del subcomponente; tolerancia al estrés, $(p \leq 0,01)$, lo que significa que a mayor nivel del componente manejo del estrés y de su subcomponente, mayor será el rendimiento académico de los estudiantes de la carrera profesional de Farmacia y Bioquímica de la Universidad Peruana Los Andes, a excepción del subcomponente control de impulsos cuyos 
resultados fueron no significativos $(p \geq 0,05)$.

En relación al componente estado de ánimo con el rendimiento académico se encontró que existe correlación significativa $(p \leq 0,01)$, así como de los subcomponentes, felicidad y optimismo $(p \leq 0,01)$, de los estudiantes de la carrera profesional de Farmacia y Bioquímica de la Universidad Peruana Los Andes lo que significa que a mayor nivel del componente estado de ánimo y de sus subcomponentes mayor será el rendimiento académico.

En relación a las teorías propuestas por Gardner (17), Salovey y Mayer (1), Sternberg (13) y Goleman (2), ha adquirido importancia la llamada inteligencia emocional al reconocerse la necesidad de contar con habilidades emocionales que repercutan en el rendimiento académico y el desempeño profesional.

\section{Agradecimientos:}

A Dios nuestro Señor y a la Virgen María Auxiliadora por guiarme en este camino de crecimiento personal y profesional. A mis compañeros de trabajo autoridades, docentes y personal administrativo de la Universidad Peruana "Los Andes". A mi asesor de Tesis, Dr. Oseda Lazo Máximo, por su colaboración para el éxito de la presente investigación.

\section{REFERENCIAS BIBLIOGRÁFICAS}

1. Salovey P, Mayer J. "Inteligencia Emocional"; 1990. Disponible en: http://www.inteligencia-emocional.org/ actividades/inteligencia_exitosa.htm.

2. Goleman D. La Inteligencia Emocional. 1ํe ed. Buenos Aires: Kairós; 1995.

3. Sánchez $H$, Reyes C. Metodología y diseños en investigación científica. Lima: Visión Universitaria; 2006

4. BarOn (1997) cit. por Ugarriza, N (2001). La Evaluación de la Inteligencia Emocional a través del Inventario de BarOn (ICE) en una muestra de Lima Metropolitana. Universidad de Lima. Lima Perú.

5. Ugarriza N, Pajarez L. Ugarriza N. La evaluación de la inteligencia emocional a través del inventario de BarOn (I-CE) en una muestra de Lima Metropolitana. Persona. 2001 ; (4): 129-160

6. Villacorta E. Inteligencia emocional y rendimiento académico en estudiantes de Medicina Humana de la Universidad Nacional de la Amazonía Peruana. lquitos. [Tesis Doctoral]; 2010. Disponible en: http://www.uap.edu.pe/Investigaciones/ Esp/Revista_12_Esp_04.pdf.

7. Valdivia J. Inteligencia emocional, estrategias de aprendizaje y rendimiento académico en estudiantes universitarios de psicología. Monterrey-México". [Tesis de Maestría]. México: Universidad Autónoma de Nuevo Señor; 2006.

8. Pérez N. Relaciones entre la inteligencia emocional y el cociente intelectual con el rendimiento académico en estudiantes universitarios. Universidad de AlicanteEspaña; 2006. Disponible en: http:// reme.uji.es/articulos/numero 22 / article6/numero\%2022\%20article $\% 20$ 6\%20RELACIONS.pdf.

9. Guerra J, et al. La relación entre la inteligencia emocional y el rendimiento académico de un estudiante de la Educación Superior. Valparaíso, Chile; 2009. Disponible en: http://www. inf.utfsm.cl/ guerra/publicaciones/ Relacion_inteligencia_emocional_y_ redimiento_academico.pdf.

10. López O. La inteligencia emocional y las estrategias de aprendizajes como predictores del rendimiento académico en estudiantes universitarios. [Tesis de Maestría]. Lima: Universidad Nacional Mayor de San Marcos; 2008. Disponible en http://www.cybertesis. edu.pe/sisbib/2008/lopez_mo/pdf/ lopez_mo.pdf.

11. Condōlo Z, Alexis J. La inteligencia emocional y rendimiento académico de los alumnos de 5to. Año de educación secundaria de la Institución Escolar María Inmaculada del distrito de San Martín de Porres Lima. [Tesis de pregrado]. Lima; 2012. Disponible en: http:// 
promo2010lenguayliteraturaunfv. blogspot.com/2010/07/inteligenciaemocional-y-rendimiento.html.

12. Bazán J. La inteligencia emocional y su relación con el nivel de logro en el área de personal social de las niñas y niños de 5 años del Jardín de Niños № 215, Trujillo, 2012. [Tesis de pregrado]. Trujillo: Universidad Cesar Vallejo; 2012. Disponible en: http://facultaddeeducacioneidiomas 1 . bligoo.pe/media/users/19/972855/ files/227730/Inteligencia_emocional.pdf.

13. Sternberg R. La inteligencia exitosa. Como una inteligencia practica y creativa determina el éxito en la vida. Barcelona. Paidos. 1997; Disponible en: http:// ocw.ub.edu/psicologia/psicologiade-leducacio-1/bloc-ii/RecursosBT_2/ Esquemas_de_los_contenidos_BT_2/ Recursos_T4_T5/5.Inteligenciaexitosa.pdf.

14. Jaimes $\bar{A}$. La Inteligencia emocional y rendimiento académico en estudiantes de la Facultad de Educación y Humanidades de la Universidad Alas Peruanas. [Tesis]. 2008; Disponible en: http://www.uap.edu. pe/Investigaciones/ Esp/Revista_09_ Esp_02.pdf.

15. Flores N. Influencia de la inteligencia general y emocional y de la capacidad de relación sobre el rendimiento académico en estudiantes de educación básica. Rioja. Madrid. 2012; Disponible en: https: //82.223.209.184/.../2012_11_07_ TFM_ESTUDIO_DEL_TRABAJO.

16. Lopez P, Salovey P, Straus R. Emotional intelligence, personality and the perceivedquality of social relationships. Personality and Individual Differences [Internet]. 2003, [fecha de consulta: 28 de noviembre de 2012]; 35(3): [641-658]. Disponible en: http://www. ingentaconnect.com/content/els/019 18869/2003/00000035/00000003/ art00242

17. GardnerH. (1983). Las inteligencias múltiples, la inteligencia emocional y el modelo de Tony Buzan. Barcelona-España. Recuperado de http://umw.inteligencia-emocional.org/ actividades/inteligencia_exitosa3.htm. 